\title{
O desafio da resistência à medicação antirretroviral em crianças infectadas pelo HIV-1
}

\section{[The challenge of antiretroviral drug resistance in HIV-1-infected children]}

\author{
Robert W. Shafer ${ }^{*}$ \\ "MD. Division of Infectious Diseases, Department of Medicine, Stanford University, Stanford, CA, \\ EUA
}

\begin{abstract}
A Organização Mundial da Saúde (OMS) estima que dois milhões de crianças com menos de 15 anos estejam infectadas pelo HIV- ${ }^{1}$. Embora a maioria das crianças infectadas viva na África subsaariana, mais de 10.000 dentre as estimadas 730.000 pessoas infectadas pelo HIV-1 no Brasil provavelmente são crianças ${ }^{1,2}$. A extensão da epidemia do HIV-1 nas crianças reflete os fatores de risco de infecção pelo HIV-1 na população adulta e a frequência com que a infecção da mãe pelo HIV-1 não é detectada e a transmissão mãe-filho não é prevenida. O tratamento do HIV-1 em crianças é mais desafiador do que em adultos e está associado a um maior risco de falha virológica.
\end{abstract}

Nesta edição do Jornal de Pediatria, Almeida et al. ${ }^{3}$ relatam a prevalência da resistência à medicação contra o HIV-1 em 47 crianças tratadas entre 2000 e 2004 em um hospital de São Paulo. Vinte e quatro dessas crianças tinham acabado de receber o diagnóstico de infecção pelo HIV-1 e não haviam recebido terapia antirretroviral (ARV) nem haviam sido expostas aos antirretrovirais durante o período perinatal. Vinte e três crianças estavam sendo tratadas com terapia ARV e apresentavam níveis detectáveis de HIV-1. O número de crianças que responderam bem ao tratamento (ou seja, que apresentavam níveis não-detectáveis de HIV-1) atendidas no mesmo hospital durante o período de estudo não foi relatado.

Entre as 24 crianças virgens de tratamento, nenhuma apresentou evidências genotípicas de susceptibilidade significativamente reduzida aos antirretrovirais. Uma criança teve um vírus com a mutação da transcriptase reversa K219N, uma mutação tipicamente selecionada pela terapia com inibidores da transcriptase reversa análogos de nucleosídeos (ITRN). Essa mutação, por si só, raramente reduz a susceptibilidade aos antirretrovirais ou o sucesso da terapia ARV. Não é possível saber se essa mutação surgiu como resultado da pressão seletiva dos antirretrovirais antes da transmissão para a criança ou como consequência de deriva genética na criança. Contudo, nem todas as crianças virgens de tratamento têm vírus suscetíveis a medicação. Na verdade, a transmissão de vírus resistentes a medicamentos

Correspondência: Robert W. Shafer, Division of Infectious Diseases, Department of Medicine, Stanford University, 94301 - Stanford, CA - EUA, rshafer@stanford.edu.

Não foram declarados conflitos de interesse associados à publicação deste editorial. 
geralmente ocorre nos filhos de mulheres que recebem terapia ARV com supressão incompleta ou profilaxia de transmissão mãe-filho ineficiente ${ }^{4-6}$.

$\mathrm{O}$ fato de as crianças virgens de tratamento terem sido diagnosticadas com média de idade de 21,5 meses é um dado preocupante. A falta de um diagnóstico mais precoce sugere que duas oportunidades foram perdidas. Em primeiro lugar, se as mães dessas crianças tivessem recebido o diagnóstico durante a gestação, é provável que a infecção pelo HIV-1 teria sido evitada $^{7,8}$. Na verdade, as infecções resultantes de transmissão mãe-filho no Brasil diminuíram de aproximadamente 2.000 por ano entre 1999 e 2003 para aproximadamente 1.100 por ano entre 2004 e 2005. Esse sucesso tem sido atribuído a uma crescente frequência na realização do teste de HIV-1 durante a gestação no país, com uma taxa de até $63 \%$ em 2006, e a um programa de profilaxia da transmissão mãe-filho modestamente bemsucedido, que reduziu o risco de transmissão mãe-filho para 7\% em $2004^{2}$.

Em segundo lugar, a ausência do diagnóstico da infecção pelo HIV-1 durante a gestação expõe o recém-nascido infectado a um alto risco de morbimortalidade durante o primeiro ano de vida - um período em que o sistema imunológico está imaturo, e o risco de progressão rápida do HIV-1 é alto ${ }^{9}$. No recente estudo sul-africano CHER, o risco de morte em bebês menores de 12 meses infectados pelo HIV-1 durante um período médio de acompanhamento de 40 semanas foi quatro vezes mais baixo (16 versus $4 \%$ ) do que em bebês randomizados para receber terapia ARV imediata em comparação com aqueles randomizados para receber terapia guiada pela contagem de $\mathrm{CD} 4^{10}$.

Entre as 23 crianças experimentadas, a maioria havia iniciado a terapia antes dos regimes de terapia antirretroviral altamente ativa (highly active antiretroviral treatment, HAART) estarem disponíveis. Evidências genotípicas da resistência do HIV-1 aos ITRN, aos inibidores da transcriptase reversa não-análogos de nucleosídeos (ITRNN) e aos inibidores da protease (IP) foram detectadas em 96, 61 e 17\%, respectivamente. As mutações de resistência mais comuns foram as mutações de resistência aos ITRN nas posições 41, 67, 184, 215 e 219 da transcriptase reversa; as mutações de resistência aos ITRNN nas posições 103 e 181 da transcriptase reversa; e as mutações de resistência aos IP nas posições 46, 54, 82 e 90 da protease.

As mutações da transcriptase reversa nas posições 41, 67, 215 e 219 são chamadas de mutações dos análogos da timidina (TAM) porque são selecionadas primariamente pelos análogos da timidina AZT e estavudina (d4T). Contudo, as TAM, especialmente a combinação de M41L e T215Y com a TAM L210W, também conferem resistência cruzada ao abacavir, à didanosina e ao tenofovir. A mutação da transcriptase reversa M184V confere alto nível de resistência fenotípica aos análogos da citidina lamivudina (3TC) e emtricitabina (FTC) e baixo nível de resistência cruzada ao abacavir e à didanosina. Apesar do alto nível de resistência fenotípica de 3TC e FTC causado pela mutação M184V, geralmente há benefícios em se incluir 3TC ou FTC em um regime terapêutico de salvação porque a M184V aumenta a susceptibilidade a AZT, d4T e tenofovir e causa uma modesta diminuição na capacidade replicativa do HIV-1. As mutações da transcriptase reversa K103N e Y181C são as duas mais comumente presentes nas mutações de resistência aos ITRNN. As mutações da protease nas posições 46, 54, 82 e 90 estão entre as mais frequentes 
nas mutações de resistência aos IP. Seis diferentes mutações de resistência aos IP na posição 54 (I54V/L/M/T/A/S) e sete diferentes mutações de resistência aos IP na posição 82 (V82A/T/F/S/L/M/C) foram relatadas. Diferentes mutações nessas duas posições geralmente apresentam efeitos divergentes na susceptibilidade dos $\mathrm{IP}^{11}$.

A grande resistência medicamentosa relatada por Almeida et al. ${ }^{3}$ é típica de crianças e adultos infectados pelo HIV-1 que iniciam a terapia com regimes não-HAART de supressão incompleta. Resultados semelhantes têm sido relatados com base em outros estudos sobre crianças fortemente tratadas no Brasil, assim como nos Estados Unidos, na França e no Reino Unido. Por exemplo, Machado et al. analisaram sequências de transcriptase reversa e protease de 37 crianças em outro hospital de São Paulo e relataram que 84, 35 e 14\% delas apresentaram vírus com mutações associadas a resistência a pelo menos uma, duas ou três classes de medicamentos, respectivamente ${ }^{12}$.

Delaugerre et al. analisaram sequências de transcriptase reversa e protease de 119 crianças em Paris entre 2001 e 2003 e relataram que 82, 32 e 27\% delas tiveram vírus com evidências genotípicas de resistência a pelo menos uma, duas e três classes de medicamentos, respectivamente, com uma resistência aos medicamentos da terceira classe significativamente mais comum nos meninos em comparação com as meninas ${ }^{13}$.

Embora o risco de falha virológica e a extensão da resistência aos antirretrovirais sejam menores entre as crianças tratadas com terapia HAART de primeira linha, tanto a falha virológica quanto a resistência também são problemas frequentes nessas crianças. Apesar da defesa imunológica contra o HIV-1 melhorar após o primeiro ano de vida ${ }^{14}$, a terapia ARV continua sendo desafiadora devido à disponibilidade de menos drogas, às formulações menos convenientes, à farmacocinética pouco previsível e aos maiores obstáculos à adesão. $\mathrm{O}$ risco de falha da terapia ARV parece ser mais alto nas crianças do que nos adultos. Por exemplo, em um grupo de 134 crianças da Abidjan, Costa do Marfim, que receberam dois ITRN + um ITRNN ou nelfinavir entre 1998 e 2003, 44\% tiveram falha virológica. De maneira semelhante, de 2004 a 2005, foi detectada falha virológica em $26 \%$ de 250 crianças comparadas com $14 \%$ de 526 adultos tratados por 6 meses com d4T + 3TC + nevirapina ou AZT + 3TC + efavirenz em uma clínica universitária em Kampala, Uganda. A falha virológica foi significativamente mais alta no sexo masculino do que no feminino, em crianças com uma porcentagem de CD4 $<5$ e em crianças sendo tratadas com a combinação de $\mathrm{d} 4 \mathrm{~T}+3 \mathrm{TC}+$ nevirapina. Entre 212 crianças cambojanas tratadas com uma das três combinações em dose fixa de dois ITRN mais um ITRNN por 12 meses entre 2003 e 2005, detectou-se viremia persistente em $19 \%{ }^{15}$. Nesses estudos, a falha virológica quase sempre esteve acompanhada do aparecimento de resistência aos ITRN e aos ITRNN.

Os subtipos B, C e F são os subtipos de HIV-1 mais comuns no Brasil. Almeida et al. relatam que 18 das 23 crianças foram infectadas com vírus do subtipo B. Portanto, não é surpreendente que as mutações da transcriptase reversa e da protease que esses autores relatam fossem típicas daquelas observadas em outros vírus do subtipo B resistentes no Brasi1 $^{16-19}$. Por outro lado, certas mutações, tais como K20T, V82L, N88S e L89V, podem ser mais comuns nos vírus do subtipo $\mathrm{F}$ no Brasil ${ }^{20,21}$, enquanto leves variações no padrão das mutações de resistência aos IP têm sido descritas em vírus do subtipo C no Brasil ${ }^{22,23}$. 
O Programa Nacional de DST e AIDS do Brasil, que tem coordenado a reação à epidemia do HIV, oferece medicamentos genéricos para tratamento de primeira linha e medicamentos com patente protegida para tratamento de segunda linha ${ }^{24}$. Em 2006, estima-se que 180.000 indivíduos infectados pelo HIV-1 estivessem recebendo tratamento no Brasil, o que representa aproximadamente $90 \%$ dos pacientes para os quais o tratamento foi indicado ${ }^{2,24}$. Indivíduos fortemente tratados, tais como as crianças descritas no estudo de Almeida et al., irão aumentar os custos do programa de tratamento do Brasil, já que é provável que um ou mais dos antirretrovirais recentemente aprovados, tais como os IP darunavir e tipranavir, o ITRNN etravirina, o inibidor de integrase raltegravir e os inibidores de entrada enfuvirtida e maraviroc serão necessários pra os pacientes com maior experiência de terapia ARV e com os níveis mais altos de resistência medicamentosa. O uso sensato desses medicamentos mais recentes sob supervisão criteriosa, para garantir adesão, e esforços crescentes para prevenir a transmissão mãe-filho e o surgimento de falha virológica durante a HAART inicial são necessários para manter a saúde das crianças infectadas pelo HIV-1 e minimizar novas infecções.

\section{Referências}

1. Joint United Nations Programme on HIV/AIDS (UNAIDS). Report on the Global HIV Epidemic. 2008. http://www.unaids.org/en/KnowledgeCentre/HIVData/GlobalReport/2008/2008_G Acesso: 05/03/2008

2. Brazilian Ministry of Health Health Surveillance Secretariat National Programme on STD and AIDS Targets and commitments made by the member-states at the United Nations. General Assembly Special Session on HIV/AIDS. UNGASS - HIV/AIDS. Brazilian Response 2005/2007 Country Progress Report. http://data.unaids.org/pub/Report/2008/ brazil_2008_country_progress_report_en.pdf. Acesso: 05/03/2008

3. Almeida FJ, Berezin EN, Rodrigues R, Sáfadi MA, Arnoni MV, Oliveira C, et al. Diversity and prevalence of antiretroviral genotypic resistance mutations among HIV-1-infected children. J Pediatr (Rio J). 2009; 85(2):104-9. [PubMed: 19319446]

4. Eshleman SH, Hoover DR, Chen S, Hudelson SE, Guay LA, Mwatha A, et al. Resistance after single-dose nevirapine prophylaxis emerges in a high proportion of Malawian newborns. Aids. 2005; 19:2167-9. [PubMed: 16284468]

5. Vignoles M, Barboni G, Agosti MR, Quarleri J, García MK, Giraudi V, et al. High frequency of primary mutations associated with antiretroviral drug resistance in recently diagnosed HIV-infected children. Antivir Ther. 2007; 12:1133-7. [PubMed: 18018772]

6. Persaud D, Palumbo P, Ziemniak C, Chen J, Ray SC, Hughes M, et al. Early archiving and predominance of nonnucleoside reverse transcriptase inhibitor-resistant HIV-1 among recently infected infants born in the United States. J Infect Dis. 2007; 195:1402-10. [PubMed: 17436219]

7. Succi RC, Figueiredo EN, Zanatta Lde C, Peixe MB, Rossi MB, Vianna LA. Evaluation of prenatal care at basic health units in the city of Sao Paulo. Rev Lat Am Enfermagem. 2008; 16:986-92. [PubMed: 19229401]

8. Stringer EM, Chi BH, Chintu N, Creek TL, Ekouevi DK, Coetzee D, et al. Monitoring effectiveness of programmes to prevent mother-to-child HIV transmission in lower-income countries. Bull World Health Organ. 2008; 86:57-62. [PubMed: 18235891]

9. Bolton-Moore C, Mubiana-Mbewe M, Cantrell RA, Chintu N, Stringer EM, Chi BH, et al. Clinical outcomes and CD4 cell response in children receiving antiretroviral therapy at primary health care facilities in Zambia. JAMA. 2007; 298:1888-99. [PubMed: 17954540]

10. Violari A, Cotton MF, Gibb DM, Babiker AG, Steyn J, Madhi SA, et al. Early antiretroviral therapy and mortality among HIV-infected infants. N Engl J Med. 2008; 359:2233-44. [PubMed: 19020325] 
11. Shafer RW, Schapiro JM. HIV-1 drug resistance mutations: an updated framework for the second decade of HAART. AIDS Rev. 2008; 10:67-84. [PubMed: 18615118]

12. Machado DM, Fernandes SC, Succi RC, Babiker AG, Steyn J, Madhi SA, et al. Analysis of HIVtype 1 protease and reverse transcriptase in Brazilian children failing highly active antiretroviral therapy (HAART). Rev Inst Med Trop Sao Paulo. 2005; 47:1-5. [PubMed: 15729467]

13. Delaugerre C, Warszawski J, Chaix ML, Veber F, Macassa E, Buseyne F, et al. Prevalence and risk factors associated with antiretroviral resistance in HIV-1-infected children. J Med Virol. 2007; 79:1261-9. [PubMed: 17607781]

14. Leal E, Janini M, Diaz RS. Selective pressures of human immunodeficiency virus type 1 (HIV-1) during pediatric infection. Infect Genet Evol. 2007; 7:694-707. [PubMed: 17719854]

15. Janssens B, Raleigh B, Soeung S, Akao K, Te V, Gupta J, et al. Effectiveness of highly active antiretroviral therapy in HIV-positive children: evaluation at 12 months in a routine program in Cambodia. Pediatrics. 2007; 120:e1134-40. [PubMed: 17954553]

16. Couto-Fernandez JC, Silva-de-Jesus C, Veloso VG, Rachid M, Gracie RS, Chequer-Fernandez SL, et al. Human immunodeficiency virus type 1 (HIV-1) genotyping in Rio de Janeiro, Brazil: assessing subtype and drug-resistance associated mutations in HIV-1 infected individuals failing highly active antiretroviral therapy. Mem Inst Oswaldo Cruz. 2005; 100:73-8. [PubMed: 15867968]

17. Cavalcanti AM, Lacerda HR, Brito AM, Pereira S, Medeiros D, Oliveira S. Antiretroviral resistance in individuals presenting therapeutic failure and subtypes of the human immunodeficiency virus type 1 in the Northeast Region of Brazil. Mem Inst Oswaldo Cruz. 2007; 102:785-92. [PubMed: 17992369]

18. de Sa-Filho DJ, Soares Mda S, Candido V, Gagliani LH, Cavaliere E, Diaz RS, et al. HIV type 1 pol gene diversity and antiretroviral drug resistance mutations in Santos, Brazil. AIDS Res Hum Retroviruses. 2008; 24:347-53. [PubMed: 18327988]

19. Eyer-Silva WA, Couto-Fernandez JC, Silva-de-Jesus C, Morgado MG. Prevalence of HIV type 1 drug resistance mutations in treatment-naive and experienced patients from resource-limited settings with universal access to antiretroviral therapy: a survey in two small Brazilian cities. Mem Inst Oswaldo Cruz. 2008; 103:143-9. [PubMed: 18425266]

20. Calazans A, Brindeiro R, Brindeiro P, Verli H, Arruda MB, Gonzalez LM, et al. Low accumulation of L90M in protease from subtype F HIV-1 with resistance to protease inhibitors is caused by the L89M polymorphism. J Infect Dis. 2005; 191:1961-70. [PubMed: 15871131]

21. Dumans AT, Barreto CC, Santos AF, Arruda M, Sousa TM, Machado ES, et al. Distinct resistance mutation and polymorphism acquisition in HIV-1 protease of subtypes B and F1 from children and adult patients under virological failure. Infect Genet Evol. 2009; 9:62-70. [PubMed: 18992847]

22. Gonzalez LM, Brindeiro RM, Aguiar RS, Pereira HS, Abreu SM, Soares MA, et al. Impact of nelfinavir resistance mutations on in vitro phenotype, fitness, and replication capacity of human immunodeficiency virus type 1 with subtype B and C proteases. Antimicrob Agents Chemother. 2004; 48:3552-5. [PubMed: 15328124]

23. Soares EA, Santos AF, Sousa TM, Sprinz E, Martinez AM, Silveira J, et al. Differential drug resistance acquisition in HIV-1 of subtypes B and C. PLoS ONE. 2007; 2:e730. [PubMed: 17710130]

24. Greco DB, Simao M. Brazilian policy of universal access to AIDS treatment: sustainability challenges and perspectives. Aids. 2007; 21(Suppl 4):S37-45. [PubMed: 17620751] 